\title{
Drag of Bodies with Arbitrary Gas-surface Accommodation in a Free-molecule Flow
}

\author{
I. Kuščer * and H. Lang \\ Max-Planck-Institut für Strömungsforschung, Göttingen \\ (Z. Naturforsch. 28 a, 1468 -1471 [1973] ; received 12 April 1973)
}

\begin{abstract}
A body of arbitrary convex shape and uniform temperature is thought to be placed in a freemolecule gas flow. In the near-equilibrium regime the four Knudsen accommodation coefficients suffice to express the drag and absorbed heat flux. Extensions to higher speeds require more information - or assumptions - on the gas-surface interaction, as is made clear by the case with $\mathrm{Ma} \gg 1$.
\end{abstract}

\section{Introduction}

Expressions for the drag exerted by a gas upon convex bodies small compared to the mean free path have been derived many times, though always under one or another restriction. Epstein ${ }^{1}$ considered slowly moving spheres, and Waldmann ${ }^{2}$ extended his results to bodies of arbitrary convex shape. Higher speeds were admitted by several authors ${ }^{3}$, who also investigated heat exchange and other effects. In most of this work either specular reflection or perfect accommodation was assumed, or a combination of both - i. e. Maxwell's boundary condition. This is equivalent to assuming all accommodation coefficients as being equal. A more flexible model involving two parameters was considered by Cercignani and Lampis ${ }^{4}$.

Only in a recent investigation for spherical particles did Williams ${ }^{5}$ permit arbitrary gas-surface scattering kernels $P\left(\boldsymbol{v}^{\prime} \rightarrow \boldsymbol{v}\right)$. However, because realistic data for such kernels are lacking, the final calculations were still carried out for simplified models, and the role of the accommodation coefficients was not completely clarified.

The principal aim of the present contribution is to supplement Williams' work by showing (Sect. 2) that in the near-equilibrium regime $(\mathrm{Ma} \ll 1$, small temperature difference), drag and heat flow can be evaluated in terms of the four Knudsen accommodation coefficients, without any detailed knowledge of the scattering law and without any restrictive assumptions. There will be no need for specialization to spheres, since at little cost bodies with arbitrary convex shape can be admitted. No such concise results can be obtained for higher speeds and temper-

* Permanent address: Department of Physics, University of Ljubljana, Yugoslavia. ature differences, except with crude simplifying assumptions (Section 3).

\section{Near-Equilibrium Regime}

We attach the coordinate system to the body and assume a drifting Maxwellian distribution $f^{-}$for the incident gas molecules, with a drift velocity $\boldsymbol{u}$ and a temperature $T=T_{0}+\Delta T$ different from that of the body $\left(T_{0}\right)$. Both temperatures shall be uniform. To simplify the writing, we only consider a monatomic gas, although this restriction is in no way essential. If $m u^{2} / 2 k T_{0} \ll 1$ and $\Delta T / T_{0} \ll 1$, we may use the approximation

$$
\begin{aligned}
f^{-}(\boldsymbol{v}) & =f^{0}(\boldsymbol{v}) \\
& \times\left[1+\frac{m}{2 k T_{0}} 2 \boldsymbol{u} \cdot \boldsymbol{v}+\frac{\Delta T}{T_{0}}\left(\frac{m v^{2}}{2 k T_{0}}-\frac{3}{2}\right)\right],
\end{aligned}
$$

where $f^{0}$ is a Maxwellian in equilibrium with the body and corresponding to the same density as implied in $f^{-}$.

The distribution $f^{+}$of the scattered molecules in the immediate neighborhood of a surface element with the outward unit normal $\boldsymbol{n}$ is related to $f^{-}$ through

$$
v_{\mathrm{n}} f^{+}(\boldsymbol{v}) \underset{v_{\mathrm{n}}{ }^{\prime}<0}{=\int}\left|v_{\mathrm{n}}{ }^{\prime}\right| f^{-}\left(\boldsymbol{v}^{\prime}\right) P\left(\boldsymbol{v}^{\prime} \rightarrow \boldsymbol{v}\right) \mathrm{d}^{3} v^{\prime},
$$

with $v_{\mathrm{n}}=\boldsymbol{v} \cdot \boldsymbol{n}, v_{\mathrm{n}}{ }^{\prime}=\boldsymbol{v}^{\prime} \cdot \boldsymbol{n}$. We substitute Eq. (1) and call upon the reciprocity (detailed balance) relation ${ }^{6-8}$,

$\left|v_{\mathrm{n}}{ }^{\prime}\right| f^{0}\left(\boldsymbol{v}^{\prime}\right) P\left(\boldsymbol{v}^{\prime} \rightarrow \boldsymbol{v}\right)=v_{\mathrm{n}} f^{0}(\boldsymbol{v}) P\left(-\boldsymbol{v} \rightarrow-\boldsymbol{v}^{\prime}\right)$.

This results in

$f^{+}(\boldsymbol{v})=f^{0}(\boldsymbol{v})$

$\times\left[1+\frac{m}{2 k T_{0}} 2 \boldsymbol{u}_{\mathrm{R}} \hat{P} \boldsymbol{v}+\frac{\Delta T}{T_{0}}\left(\frac{m}{2 k T_{0}} \hat{P} v^{2}-\frac{3}{2}\right)\right]$, 
where the operator $\hat{P}$ is defined as follows,

$$
\hat{P} Q(\boldsymbol{v}) \underset{v_{\mathrm{n}}^{\prime}>0}{=\int} P\left(\boldsymbol{v}_{\mathrm{R}} \rightarrow \boldsymbol{v}^{\prime}\right) Q\left(\boldsymbol{v}^{\prime}\right) \mathrm{d}^{3} v^{\prime},
$$

with $\boldsymbol{v}_{\mathrm{R}}=-\boldsymbol{v}_{\mathrm{n}}+\boldsymbol{v}_{\mathrm{t}}$ if $\boldsymbol{v}_{\mathrm{n}}$ and $\boldsymbol{v}_{\mathrm{t}}$ are the normal and tangential components of $\boldsymbol{v}$, respectively. The surface is assumed as being isotropic, i. e., $P\left(\boldsymbol{v}^{\prime} \rightarrow \boldsymbol{v}\right)$ is invariant against rotation around $\boldsymbol{n}$.

By calculating the incident and reflected fluxes of tangential and normal momentum, and of energy, we determine the tangential and normal stress and the absorbed heat flux as

$$
\begin{gathered}
\boldsymbol{\tau}=\frac{1}{4} \varrho v_{0} \alpha_{22} \boldsymbol{u}_{\mathrm{t}}, \\
\boldsymbol{p}=-\left[p-\frac{1}{4} \varrho v_{0}\left(\zeta+\alpha_{22}\right) u_{\mathrm{n}}-\frac{1}{4} p \alpha_{14}\right. \\
\left.\cdot\left(\Delta T / T_{0}\right)\right] \boldsymbol{n}, \\
q=\frac{1}{4} p\left[-\alpha_{14} u_{\mathrm{n}}+2 v_{0} \alpha_{44}\left(\Delta T / T_{0}\right)\right],
\end{gathered}
$$

where $p=n k T$ and $\varrho=n m$ are the pressure and density of the gas, and $v_{0}=\left(8 k T_{0} / \pi m\right)^{1 / 2}$. The Knudsen accommodation coefficients appear here ${ }^{9-11}$ i. e. those for normal momentum $\left(\alpha_{11}\right)$, tangential momentum $\left(\alpha_{22}\right)$ and energy $\left(\alpha_{44}\right)$, and the radiometric coefficient $\left(\alpha_{14}\right)$. Their definition is

$$
\alpha_{i j}=\frac{\left[\left\langle Q_{i} Q_{j}\right\rangle-\left\langle Q_{i} \hat{P} Q_{j}\right\rangle\right]}{\left[\left\langle Q_{i} Q_{j}\right\rangle-\left\langle Q_{i}\right\rangle\left\langle Q_{j}\right\rangle\right]},
$$

with $Q_{1}=v_{\mathrm{n}}, Q_{2}=v_{\mathrm{t}}$ and $Q_{4}=v^{2}$. The triangular brackets indicate averages over a Maxwellian flux corresponding to the temperature of the body,

$$
\langle\ldots\rangle=\frac{2}{\pi}\left(\frac{m}{2 k T_{0}}\right)_{v_{\mathrm{n}}>0}^{2} \int_{>}(\ldots) v_{n} \exp \left\{-\frac{m v^{2}}{2 k T_{0}}\right\} \mathrm{d}^{3} v .
$$

For convenience we have also introduced the coefficient

$$
\zeta=4-2\left(1-\frac{1}{4} \pi\right) \alpha_{11}-\alpha_{22},
$$

whose values are limited by $4>\zeta>1+\frac{1}{2} \pi=2.57$ if both accommodation coefficients are between 0 and 1 .

Equation (4) and the term involving $\alpha_{44}$ in Eq. (6) are well known. The last one in Eq. (5) has already been derived by Knudsen ${ }^{12}$ as representing the free-molecular radiometer pressure, i. e., the excess pressure caused by the temperature difference between a collisionless gas and a surface. In Eq. (6) we notice a conjugate term describing the excess heat flux caused by the gas flow. All three expressions coincide with the leading terms derived from the formulas of Schaaf and Chambré ${ }^{3}$, provided that we assume $\alpha_{14}=\alpha_{11}$ in Eq. (5), and $\alpha_{14}=\alpha_{44}$ in Equation (6).

Integration over the surface (total area $A$ ) yields the final results for the force upon the body and the total heat flow,

$$
\begin{aligned}
& \boldsymbol{F}=\frac{1}{4} \varrho v_{0}\left[\alpha_{22} \boldsymbol{u}+\zeta \overline{(\boldsymbol{n} \otimes \boldsymbol{n})} \cdot \boldsymbol{u}\right] A, \\
& \Phi=\frac{1}{2} p v_{0} \alpha_{44}\left(\Delta T / T_{0}\right) A .
\end{aligned}
$$

The factor $(\boldsymbol{n} \otimes \boldsymbol{n})$ in Eq. (8) represents the average over the surface of the body of a tensor product, with the components $n_{i} n_{j}, i, j=1,2,3$. We notice that both radiometric effects have dropped out from the above first-order approximations.

For a non-spherical body the drag varies with orientation, and its direction may deviate from that of the flow. Yet, if the body has a center of symmetry, the torque with respect to that center clearly vanishes.

If the body is rotationally symmetric and the axis is parallel to $\boldsymbol{u}$, expression (8) simplifies to

$$
\boldsymbol{F}=\frac{1}{4} \varrho v_{0}\left(\alpha_{22}+\zeta \overline{\cos ^{2} \vartheta}\right) \boldsymbol{u} A,
$$

with $\vartheta$ representing the angle of incidence of the flow $(u \cos \vartheta=-\boldsymbol{n} \cdot \boldsymbol{u})$. For a rotational ellipsoid oriented in the direction of flow $\overline{\cos ^{2} \vartheta}$ can be expressed in an elementary way, and in the limit of a strongly elongated ellipsoid we find $\overline{\cos ^{2} \vartheta} \approx(b / a)^{2}$. The same result also approximately holds for a double cone inscribed to the ellipsoid.

We may introduce the cross section $A_{\perp}$ of the body, as viewed in the direction $\boldsymbol{u}$, and study the dimensionless coefficient $C_{1}$ in a modified form of Equation (10),

$$
F=C_{1} p A_{\perp} \mathrm{Ma}
$$

where $\mathrm{Ma}$ is the Mach number. Since $\zeta$ stays finite, a question about the lower bound of $C_{1}$ arises. The example with the elongated ellipsoid indicates that $C_{1}$ could be made arbitrarily small, thus giving a "perfect slip", if only surfaces with sufficiently small $\alpha_{22}$ were available. Though this is hardly more than wishful thinking, it is instructive to find out that for the optimal ellipsoid (or double cone) the minimum of $C_{1}$ is of the order of $\left(\zeta \alpha_{22}\right)^{1 / 2}$.

In case of a sphere $\left.\overline{\left(\cos ^{2} \vartheta\right.}=\frac{1}{3}\right)$, and if $\alpha_{11}=\alpha_{22}$, Eq. (10) agrees with Epstein and Waldmann ${ }^{2}$. On the other hand, Eq. (10) should be compared with the corresponding result of Williams ${ }^{5}$. A relationship of the first-order Legendre coefficient of his global scattering kernel to the accommodation coef- 
ficients is derived thereby,

$$
\begin{gathered}
\frac{4}{3}\left[1-\frac{1}{\pi r^{2}}\left(\frac{m}{2 k T_{0}}\right)^{3} \int_{0}^{\infty} \int_{0}^{\infty} v^{3} v^{\prime 3} \exp \left\{-\frac{m v^{\prime 2}}{2 k T_{0}}\right\}\right. \\
\left.\times \sigma_{1}\left(v^{\prime} \rightarrow v\right) \mathrm{d} v \mathrm{~d} v^{\prime}\right]=\alpha_{22}+\frac{1}{3} \zeta
\end{gathered}
$$

In the same way, Eq. (9) leads to

$$
\begin{array}{r}
3-\frac{1}{\pi r^{2}}\left(\begin{array}{c}
m \\
2 k T_{0}
\end{array}\right)^{4} \int_{0}^{\infty} \int_{0}^{\infty} v^{4} v^{\prime 4} \exp \left\{-\frac{m v^{\prime 2}}{2 k T_{0}}\right\} \\
\times \sigma_{0}\left(v^{\prime} \rightarrow v\right) \mathrm{d} v \mathrm{~d} v^{\prime}=\alpha_{44} .
\end{array}
$$

With some effort, both indentities can also be verified in a direct manner.

\section{Hypersonic Limit}

At intermediate speeds and temperature differences it appears possible to extend the previous approach by proceeding up to quadratic terms in the Taylor expansion (1) of $f^{-}$. Improved versions of Eqs. (4) - (6), (8), (9) can be derived thereby, with the additional terms proportional to $u^{2}$, $\left(\Delta T / T_{0}\right)^{2}$, and $u \Delta T / T_{0}$. The corresponding coefficients may be expressed by "second-order" accommodation coefficients ${ }^{11,13}$, which are introduced by admitting further polynomials $Q_{i}(\boldsymbol{v})$ (those known from Grad's 13-moments method, supplemented by $v^{4}$ ) in the definition (7). Only if some of the accommodation coefficients are assumed as equal, will such a result coincide with quadratic approximations to the formulas of Schaaf and Chambré. On the other hand, a comparison to the expressions of Williams should reveal further identities for the global scattering kernel.

For the drag of an axially symmetric body, with the axis in the direction of flow, and with $\Delta T=0$, a continuation of this procedure should lead to

$$
F=p A_{\perp}\left(C_{1} \mathrm{Ma}+C_{2} \mathrm{Ma}^{2}+C_{3} \mathrm{Ma}^{3}+\ldots\right) .
$$

If the body has a center of symmetry, the even terms obviously vanish, so that at least $C_{3}$ ought to be considered, and expressed in terms of "third-order" accommodation coefficients. These undoubtedly are beyond present possibilities of experimental determination, so that there is good reason to abandon such an approach altogether.

Exact results for arbitrary speeds and temperature differences can only be derived with full knowl- edge of the gas-surface scattering kernel, or alternatively by adopting models or other simplifying assumptions. By way of illustration we review the case of the hypersonic limit, $\mathrm{Ma} \rightarrow \infty$, which has already been considered by several authors ${ }^{3,14-16}$. A vanishing gas temperature is assumed which is to say that all the molecules strike the surface with the same velocity $\boldsymbol{u}$. We shall also take $T_{0}=0$, which is justified if the surface is relatively cool $\left(k T_{0} \ll \frac{1}{2} m u^{2}\right)$. Approximately such a regime is encountered by a space vehicle in the highest layers of the atmosphere, where radiation cooling still outweighs frictional heating, or in experiments with rarefied free jets.

Now the so called beam accommodation coefficients ${ }^{11}$ must be used, which are functions of the incident velocity. Their definition is

$\alpha_{\mathrm{i}}(u, \cos \vartheta)=1-$

$$
\left[\hat{P} Q_{\mathrm{i}}\left(\boldsymbol{u}_{\mathrm{R}}\right)-\left\langle Q_{\mathrm{i}}\right\rangle\right] /\left[Q_{\mathrm{i}}\left(\boldsymbol{u}_{\mathrm{R}}\right)-\left\langle Q_{\mathrm{i}}\right\rangle\right],
$$

which in the present case reduces to $\alpha_{\mathrm{i}}(u, \cos \vartheta)$ $=1-\hat{P} Q_{\mathrm{i}} / Q_{\mathrm{i}}$ because the $\left\langle Q_{\mathrm{i}}\right\rangle$ vanish for $T_{0}=0$.

In the same way as before we find (for $\cos \vartheta>0$ )

$$
\begin{aligned}
& \quad \boldsymbol{p}=-\varrho u_{\mathrm{n}}^{2}\left[2-\alpha_{1}(u, \cos \vartheta)\right] \boldsymbol{n}, \\
& \boldsymbol{\tau}=\varrho\left|u_{\mathrm{n}}\right| \boldsymbol{u}_{\mathrm{t}} \alpha_{2}(u, \cos \vartheta), \\
& q=\frac{1}{2} \varrho u^{2}\left|u_{\mathrm{n}}\right| \alpha_{4}(u, \cos \vartheta),
\end{aligned}
$$

Integration over the surface of the body now also affects the $\alpha_{\mathrm{i}}$ because $\cos \vartheta$ varies with the orientation of the surface normal. Since insufficient data about the coefficients $\alpha_{\mathrm{i}}(u, \cos \vartheta)$ are available, it is customary to assume that they are constants in the range of interest - though this is hardly ever the case. Under such assumption the integration leads to the following result for an axially symmetric body with the axis parallel to $\boldsymbol{u}$,

$$
\begin{aligned}
F & =C_{\mathrm{D}} \frac{1}{2} \varrho u^{2} A_{\perp}, \\
C_{\mathrm{D}} & =2\left[\alpha_{2}+\left(2-\alpha_{1}-\alpha_{2}\right) \overline{\cos ^{3} \vartheta} / \overline{\cos \vartheta},\right. \\
\Phi & =\frac{1}{2} \varrho u^{3} A_{\perp} \alpha_{4} .
\end{aligned}
$$

The averages are now taken over the part of the surface exposed to the gas flow. Actually, if the $\alpha_{\mathrm{i}}$ are not constants, more complicated averages than shown above are involved, such as $\overline{\left(2-\alpha_{1}-\alpha_{2}\right) \cos ^{3} \vartheta}$.

Again, in the case of a sphere $\overline{(\cos \vartheta}=\frac{1}{2}$, $\cos ^{3} \vartheta=\frac{1}{4}$ ) we obtain from Eq. (17)

$$
C_{\mathrm{D}}=2-\alpha_{1}+\alpha_{2},
$$

which has been given by several authors ${ }^{14,16}$. 
We may repeat the admittedly academic question about the perfect slip. For $\alpha_{2} \ll 1$ the optimal ellipsoid as determined from Eq. (17), is found to give $C_{\mathrm{D}, \min } \approx 8 \pi\left[\alpha_{2}\left(2-\alpha_{1}\right)\right]^{1 / 2}$.

1 P. S. Epstein, Phys. Rev. 23, 710 [1924].

2 L. Waldmann, Z. Naturforsch. 14 a, 589 [1959].

3 S. A. Schaaf and P. L. Chambré, in "Fundamentals of Gas Dynamics", ed. by H. W. Emmons (High Speed Aero-dynamics and Jet Propulsion, Vol. IV), Princeton Univ. Press 1958; S A. Schaaf, in "Encyclopedia of Physics", ed. by S. Flügge, Springer, Berlin 1963. See also references quoted in these two articles.

${ }^{4}$ C. Cercignani and M. Lampis, Entropie 44, 40 [1972]; Z. angew. Math. Phys. 23, 713 [1972] ; M. Lampis, Rarefied Gas Dynamics, 8th Symposium (to be published).

5 M. M. R. Williams, Z. Naturforsch. 27 a, 1798, 1804 [1972].

${ }^{6}$ C. Cercignani, Mathematical Methods in Kinetic Theory, Plenum Press, New York 1969.

7 E. P. Wenaas, J. Chem. Phys. 54, 376 [1971].

8 I. Kuščer, Surface Sci. 25, 225 [1971].

\section{Acknowledgement}

This work originated during a visit of one of the authors (I. K.) to the Max-Planck-Institute. He wishes to thank Professor E.-A. Müller and Dr. K. Kraemer for their kind hospitality.

${ }^{9}$ S. F. Shen and I. Kuščer, Rarefied Gas Dynamics, 7th Symposium (to be published).

10 I. Kuščer, J. Možina, and F. Križanič, Rarefied Gas Dynamics, 7th Symposium (to be published).

11 I. Kuščer (to be published).

12 M. Knudsen, Kgl. Danske Vidensk. Selsk. Math.-fys. Meddelelser 11, No. 1 [1930]; M. Knudsen, The Kinetic Theory of Gases, Methuen, London 1952.

13 J. Možina, N. J. McCormick, and I. Kuščer, Rarefied Gas Dynamics, 8th Symposium (to be published).

14 G. N. Patterson, AIAA J. 3, 577 [1965] ; UTIAS Review No. 18, Univ. of Toronto 1964.

15 D. P. Jackson and J. B. French, Rarefied Gas Dynamics, 6th Symposium 1969, p. 1119.

16 E. D. Knechtel and W. C. Pitts, Rarefied Gas Dynamics, 6th Symposium 1969, p. 1257. 